\title{
Unintended consequences
}

\author{
Samuel Hellman
}

Many practicing physicians have recently received "Important drug warning and new prescribing information" from Amgen and Ortho Biotech with regard to formulations of erythropoietin. Apparently, patients receiving these agents have an increased incidence of thromboses and thromboembolic events (Bohlius $\mathrm{J}$ et al. [2006] J Natl Cancer Inst 98: 708-714). I suggest that a possible cause of some of these problems is altered hematopoietic stem-cell differentiation, favoring erythrocytic and thrombocytic pathways at the expense of granulopoiesis following bleeding or erythropoietin administration. This preferential erythropoietic differentiation was first reported in mice almost 40 years ago (Hellman S and Grate HE [1967] Nature 216: 65-66). This competition of the differentiation pathways argued strongly for the functional importance of a common hematopoietic stem cell. In these experiments, transplanted bonemarrow hematopoietic progenitor cells preferentially differentiated towards the erythrocytic pathway when administered into whole-body irradiated syngeneic recipients that had been bled or given erythropoietin. Hematopoietic stem-cell differentiation favoring erythropoiesis occurred at the expense of a concomitant diminished granulocyte response to bacterial endotoxin. This preferential differentiation toward the erythrocytic pathway is accompanied by increased platelet production, which might be the cause of the increased thromboembolic events seen with the clinical use of erythropoietin (Goodman R et al. [1977] Blood 49: 253-261). The decrease in granulocyte production associated with increased erythrocytic stimulation may lead to infectious complications, which should be looked for in patients treated with erythropoietin, especially those receiving chemotherapy or extensive radiation therapy.

\section{This preferential differentiation toward the erythrocytic pathway. . . might be the cause of the increased thromboembolic events...}

$S$ Hellman is an Advisory Board member of Nature Clinical Practice Oncology.

\section{Competing interests \\ The author declared no competing interests. \\ www.nature.com/clinicalpractice doi:10.1038/ncponc0900}

Another complication reported in patients with cancer treated with erythropoietin was increased tumor growth (Henke M [2003] Lancet 362: 1255-1260), which might be because of a better oxygen supply to the tumor. The increased tumor proliferation, however, might also be attributable to erythropoietin directly stimulating tumor cells.

In this instance, the term erythropoietin does not fully describe the activity of the agent. Such naming of agents on the basis of their first observed function may restrict our thinking with regard to the properties of the agent. The major oncologically-related effects of plateletderived growth factor relate to its angiogenic and tumor cell proliferation stimulating activities rather than to platelets. Other examples of substances with more protean activities that are named for their first described function include tumor necrosis factor and the heat shock proteins, all of which have many actions beyond those described by their names.

There are at least two lessons to be learned from these clinical results. First, it pays to review animal experiments before embarking on human administration, even if the experiments are in the old literature. While there are considerable species-related differences, one of the purposes of doing experiments in animals is to provide guidance in determining both the uses and possible untoward effects of new therapies. The older literature may be more difficult to access, but that does not limit its relevance. Second, what's in a name? The term 'erythropoietin' may not describe the universe of the activities of this biologically important substance. While clearly there were unintended consequences of erythropoietin use, they should not have been completely unexpected. 